\title{
Geogebra e Cubo de Yoshimoto: uso de recurso digital e de material didático manipulativo no cálculo do volume do Sólido de Escher
}

\author{
Olga Harumi Saito ${ }^{1}$ \\ Departamento Acadêmico de Matemática, UTFPR, Curitiba, PR \\ Ederson Marcelino da Silva ${ }^{2}$ \\ Departamento de Ensino, Colégio Militar de Curitiba (CMC), Curitiba, PR
}

Resumo. Este trabalho mostra a obra de Maurits Cornelis Escher, "Cascata", destacando o Sólido de Escher como objeto motivador para o cálculo do volume de sólidos. Utilizando o recurso digital Geogebra esse sólido, que é um dodecaedro rômbico estrelado, é construído e uma fórmula para calcular o seu volume é deduzida. Como alternativa o Cubo de Yoshimoto é apresentado, um material didático manipulável decomponível em dois Sólidos de Escher, que permite visualizar as propriedades envolvidas. Dessa forma é possível associar arte, recurso digital e material concreto no ensino de volume de sólidos.

Palavras-chave. Geometria dos Sólidos, Volume dos Sólidos, Graficador 3D, Material Concreto

\section{Introdução}

De acordo com Silveira [9], as dificuldades com o aprendizado em Matemática estão presentes no cotidiano de muitas pessoas, então é importante apresentar elementos para atrair a atenção dos estudantes quando se está ensinando um determinado conteúdo.

Os Parâmetros Curriculares Nacionais (PCNs) [1], salientam a importância do trabalho com a codificação e decodificação de desenhos e também reforçam a necessidade de desenvolvimento das habilidades de percepção espacial.

Obras de arte se apresentam como uma opção e, associadas ao uso de ferramentas como o Geogebra, têm auxiliado no ensino de conceitos e propriedades em diversos conteúdos da Matemática.

Além disso, Vieira [10] comenta que o uso de materiais didáticos manipulativos possibilitam ao aluno ter controle sobre as propriedades e operações envolvidas ao estudar assuntos como Geometria, em especial, no cálculo do volume dos sólidos.

\footnotetext{
${ }^{1}$ harumi@utfpr.edu.br

${ }^{2}$ edersonmarcelinodasilva@gmail.com
} 


\section{Sólidos Geométricos e Arte}

Apesar das pessoas verem a beleza nas construções como a obra Atomium, Figura 1(a), e a Biblioteca Nacional da Bielorrússia, Figura 1(b), conhecida como "diamante bielorusso", muitas vezes não as associam aos conceitos matemáticos envolvidos.

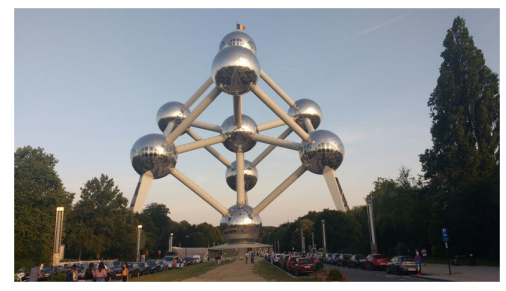

(a)

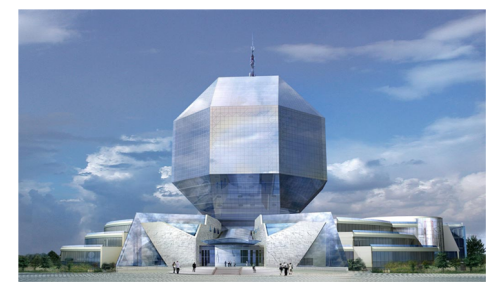

(b)

Figura 1: (a) Atomium: formato baseado em um cristal de ferro [4]; (b) Biblioteca Nacional da Bielorrussia: forma de um rombicuboctaedro [7].

Vários artistas fazem uso da geometria espacial em suas obras, seja por sua beleza ou pelas possibilidades que elas oferecem. Entre esses artistas, destaca-se Maurits Cornelis Escher.

\subsection{Sólido de Escher}

Maurits Cornelis Escher (1898-1972) foi um grande entusiasta da Matemática, mesmo não tendo muito conhecimento nessa área. Seus trabalhos apresentam muitos conceitos matemáticos que podem ser explorados, sendo uma referência para o estudo de Geometria [3]. Ele realizou diversos trabalhos em que os sólidos geométricos podem ser vistos, como na litogravura Cascata, Figura 2.

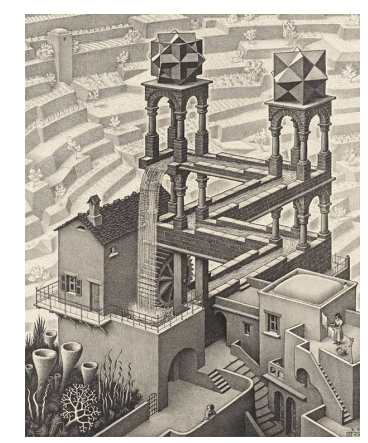

Figura 2: Litogravura Cascata [3].

Nota-se, no alto das torres, dois poliedros, o que está sobre a torre da esquerda é uma composição de três cubos e o da torre da direita é um dodecaedro rômbico estrelado que, devido a essa obra, ficou conhecido como Sólido de Escher. 


\section{Volume do Sólido de Escher}

Pela definição de arestas, faces e vértices [6], o Sólido de Escher (SE) possui 12 faces, Figura 3(b), geradas da expansão de cada uma das 12 faces dododecaedro rômbico, Figura $3(\mathrm{a})$.

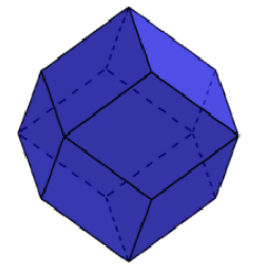

(a)

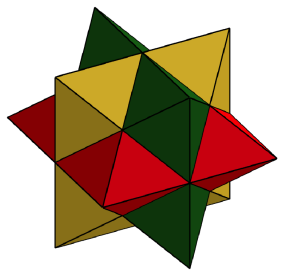

(b)

Figura 3: Sólido de Escher gerado no Geogebra: (a) dodecaedro rômbico; (b) dodecaedro rômbico estrelado - Sólido de Escher [5].

Silva [4] durante o seu trabalho de mestrado desenvolveu um tutorial no Geogebra, disponível em [5], sobre como construir o Sólido de Escher utilizando essa ferramenta.

Uma face do SE é a união de todos os triângulos contidos no mesmo plano como, por exemplo, os triângulos AEC, CGB, EFH, GHI, Figura 4(a). Ainda, os vértices do dodecaedro rômbico, como C, E, F e G são chamados de falsos vértices no Sólido de Escher. Considerando que as arestas do SE tem medida $2 a$, isto é, $A B=2 a$, consequentemente $A C=a$.

No SE há ainda outros tipos de segmentos, por exemplo $\overline{A E}$ que serão considerados de medida $b, a>b$.

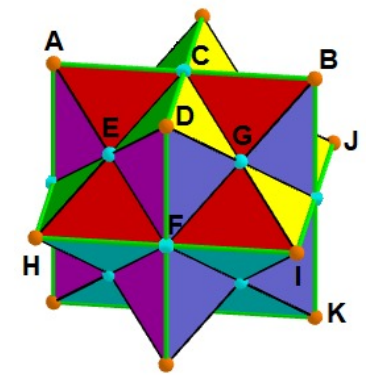

(a)

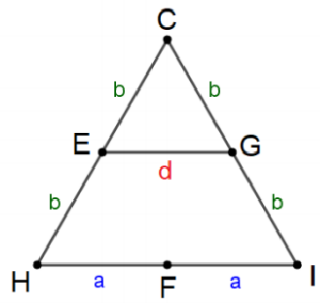

(b)

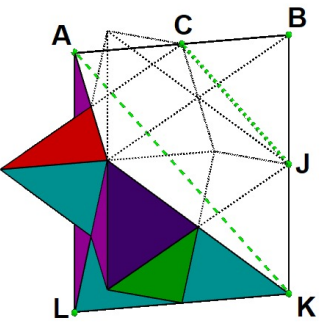

(c)

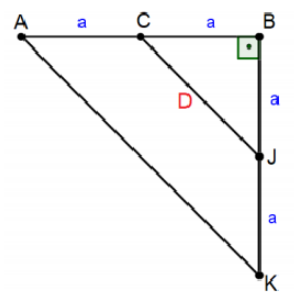

(d)

Figura 4: (a) Sólido de Escher; (b) diagonal menor d; (c) secção do Sólido de Escher; (d) diagonal maior $D[4]$.

Os segmentos $C E, E F, F G$ e $G C$, por coincidirem com as arestas dododecaedro rômbico, são chamados de falsas arestas, também com medida $b$. 
E o volume do Sólido de Escher $\left(V_{S E}\right)$ é igual ao volume dododecaedro rômbico $\left(V_{D R}\right)$ acrescido do volume de doze pirâmides $\left(V_{P 1}\right)$, ou seja:

$$
V_{S E}=V_{D R}+12 V_{P_{1}} \text {. }
$$

O triângulo $C H I$ é isósceles de base $2 a$ e lados $2 b$, Figura 4(b) e, por semelhança de triângulos $a=d$. Assim, a diagonal menor $(d)$ do losango CEFG tem a mesma medida $a$ dos maior segmento entre dois pontos do SE.

Seccionando o SE, Figura 4(c), $A B K L$ é um quadrado de lado $2 a, \overline{C J}$ é a diagonal maior $(D)$ do losango formado em $A B K$ é um triângulo isósceles, retângulo em $B$, Figura $4(\mathrm{~d})$.

Como o triângulo $A B K$ é retângulo em $B$, por ser a metade do quadrado $A B K L$, o triângulo $C B J$ também possui essas características. Utilizando o Teorema de Pitágoras, obtém-se $D=a \sqrt{2}$ (diagonal do losango). Portanto, as faces losangulares do DR têm diagonais $a$ e $a \sqrt{2}$.

Para a altura $h$ da pirâmide, Figura 5(a), utiliza-se novamente o Teorema de Pitágoras e $h=\frac{a \sqrt{2}}{2}$.

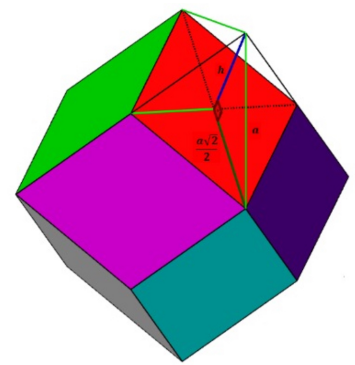

(a)

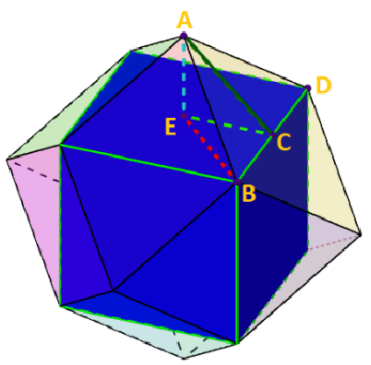

(b)

Figura 5: Dodecaedro rômbico: (a) altura $h$ da pirâmide sobre a face; (b) decomposto em um cubo e 6 pirâmides [4].

Assim, o volume de cada pirâmide $V_{P_{1}}$ é dado por:

$$
\begin{aligned}
V_{P_{1}} & =\frac{1}{3}\left(\frac{d \cdot D}{2}\right) h ; \\
V_{P_{1}} & =\frac{1}{3}\left(\frac{a \cdot a \sqrt{2}}{2}\right)\left(\frac{a \sqrt{2}}{2}\right) ; \\
V_{P_{1}} & =\frac{a^{3}}{6} .
\end{aligned}
$$

Um dodecaedro rômbico pode ser decomposto em um cubo, com arestas de mesma medida das diagonais menores das faces losangulares do DR, com 6 pirâmides quadrangulares 
em cada face, Figura 5(b).

$\mathrm{E}$, com isso o volume do $\mathrm{DR}\left(V_{D R}\right)$ equivale ao volume do cubo $\left(V_{C B}\right)$ de arestas $d=a$ e a soma do volume das seis pirâmides $\left(6 V_{P_{2}}\right)$, ou seja,

$$
\begin{aligned}
V_{D R} & =V_{C B}+6 V_{P_{2}} ; \\
V_{D R} & =a^{3}+6 \frac{1}{3}\left(a^{2} \frac{1}{2}\right) ; \\
V_{D R} & =2 a^{3} .
\end{aligned}
$$

Definido o volume do DR (3) e das pirâmides de sua estrelação (2), uma fórmula para calcular o volume do Sólido de Escher (1) é dada por:

$$
\begin{aligned}
& V_{S E}=V_{D R}+12 V_{P_{1}} \\
& V_{S E}=2 a^{3}+12 \frac{a^{3}}{6} ; \\
& V_{S E}=4 a^{3} .
\end{aligned}
$$

Assim, o volume do SE equivale ao volume de quatro cubos com arestas a que é a medida dos segmentos, entre dois pontos, de maior comprimento do SE.

No Geogebra, após a construção e através da aba Álgebra é possível visualisar o valor do volume utilizando aresta $a=2$ unidades de comprimento, Figura 6 .

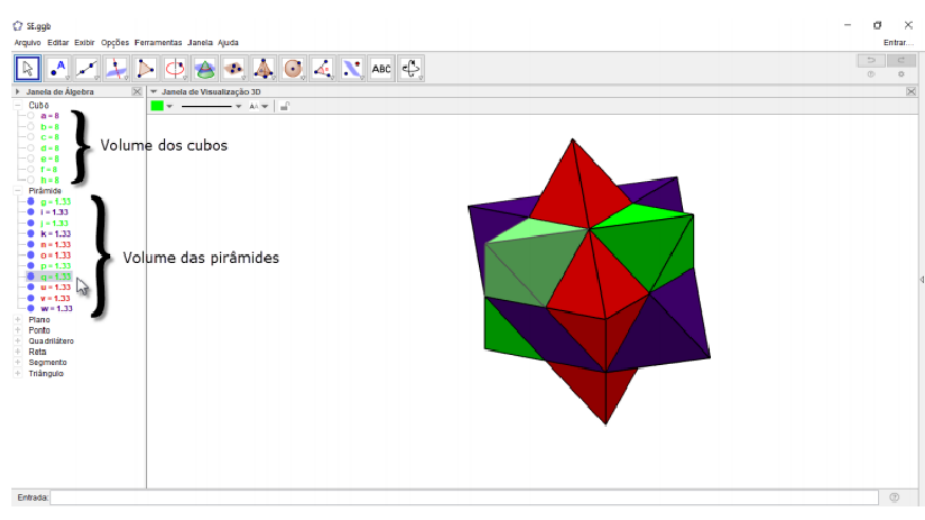

Figura 6: Sólido de Escher construído no Geogebra e detalhes da aba "Àlgebra" [5].

\section{Visualizando o volume do Sólido de Escher através do Cubo de Yoshimoto}

O Cubo de Yoshimoto é um quebra-cabeça poliédrico mecânico desenvolvido por Naoki Yoshimoto em 1970 [2]. Para a sua confecção podem ser utilizados diversos materiais como madeira, acrílico ou ainda, de uma forma mais acessível, em papel. 


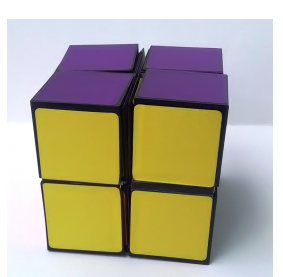

(a)

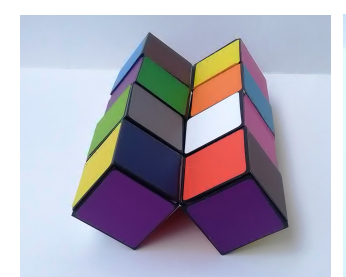

(b)

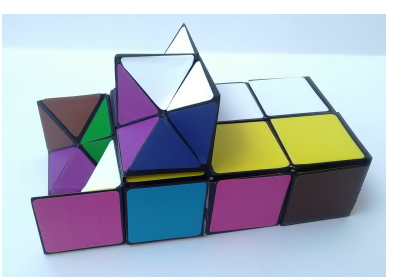

(c)

Figura 7: Quebra-cabeça poliédrico Cubo de Yoshimoto:(a) cubo fechado; (b) manipulando o cubo; (c) separando o cubo em duas partes [4].

A Figura 7(a), mostra o Cubo de Yoshimoto (CY) que é composto por 8 cubos com arestas de medida $a$ e volume $V_{C Y}=8 a^{3}$.

Manipulando-o, Figura 7(b) e (c), o Cubo de Yoshimoto pode ser separado em dois objetos com as mesmas dimensões e volumes, Figura 8(a), (b) e (c). Cada uma das duas partes pode se transformar em um Sólido de Escher, cujo volume é igual à metade do volume do Cubo de Yoshimoto, ou seja, $V_{S E}=4 a^{3}$.

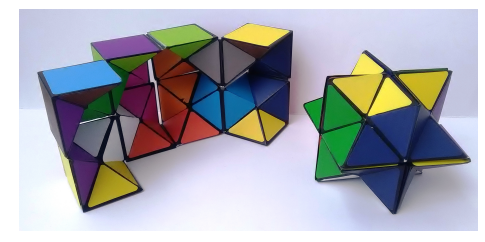

(a)

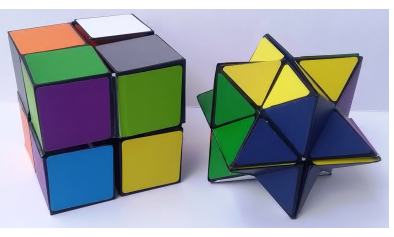

(b)

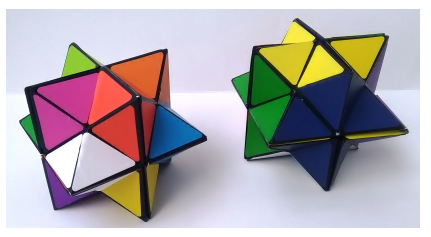

(c)

Figura 8: Quebra-cabeça poliédrico Cubo de Yoshimoto:(a) cubo separado em duas partes; (b) uma peça fechada e a outra Sólido de Escher; (c) transformando em dois Sólidos de Escher [4].

Através dessas manipulações, o estudante pode compreender e visualisar as faces do Sólido de Escher que estão no mesmo plano entre outras características e particularidades.

\section{Conclusões}

Materiais didáticos manipuláveis associados a recursos digitais possibilitam ao estudante compreender e visualizar de uma forma simples os objetos tridimensionais não se limitando a esboçá-los em duas dimensões e que muitas vezes não contribuem para resolver um determinado problema.

O Geogebra como ferramenta para construir os sólidos geométricos contribui para o entendimento dos conceitos envolvidos em seus estudos, podendo ser confirmados através de material didático manipulativo.

Trabalhar em conjunto com outras disciplinas como artes e informática quando se fala no Sólido de Escher e no Cubo de Yoshimoto possibilitam motivar o estudante. São 
materiais de fácil acesso e recomendados o seu uso sempre que possível.

\section{Agradecimentos}

À Coordenação de Aperfeiçoamento de Pessoal de Nível Superior - Brasil (CAPES) pela recomendação do PROFMAT por meio do parecer do Conselho Técnico Científico da Educação Superior e pelo incentivo financeiro - Código de Financiamento 001.

\section{Referências}

[1] Brasil. Parâmetros Curriculares Nacionais: terceiro e quarto ciclos do Ensino Fundadamental - Matemática. Brasília: MEC/SEF, 1998, http://portal.mec.gov.br/seb/ arquivos/pdf/matematica.pdf. Acesso em 12 de julho de 2018.

[2] D. Brill, Yoshimoto Cube developments. https://brilliantorigami.com/2016/01/23/yo shimoto-cube-developments. Acesso em 12 de fevereiro de 2019.

[3] Escher. M. C. Escher: The Official Website. http://www.mcescher.com. Acesso em 25 fevereiro de 2019 .

[4] E. M. da Silva. Poliedros de Arquimedes, Catalan, Kepler-Poinsot, Platão e o Sólido de Escher: contribuições para o ensino e aprendizagem de poliedros, Dissertação de Mestrado, UTFPR, Curitiba, PR, 2018.

[5] E. M. da Silva. GeoGebra Math Apps. Sólido de Escher (Dodecaedro Rômbico Estrelado). https://www.geogebra.org/m/ffevp7zt. Acesso em 25 de fevereiro de 2019.

[6] R. T. Lima, A. A. B. S. Luz, A. R. T. Góes. Poliedros estrelados: o estudo dos sólidos geométricos além dos livros didáticos. Florianópolis: GRAPHICA, 2013. https://goo.gl/jTE4qH. Acesso em 12 julho de 2018.

[7] R. Motulsky. Biblioteca Nacional da Bielorrússia. http: //www.nlb.by. Acesso em 21 de fevereiro de 2019.

[8] C. Segadas, F. R. Silva, M. Moutinho. Explorando atividades de visualização e representação de figuras no espaço. VIII Encontro Nacional de Educação Matemática - ENEM, Universidade de Pernambuco, Pernambuco, 2004. http: //www.sbembrasil.org.br/files/viii/pdf/02/MC81955154791.pdf. Acesso em 12 julho de 2018.

[9] M. R. A. da Silveira. "Matemática é Difícil": um sentido pré-construído evidenciado na fala dos alunos, Coletânea do Programa de Pós-Graduação em Educação, Porto Alegre, v. 7, n.21, p. 34-40, 1999.

[10] C. R. Vieira. Reinventando a geometria no ensino médio: uma abordagem envolvendo materiais concretos, softwares de geometria dinâmica e a Teoria de Van Hiele, Dissertação de Mestrado, Universidade Federal de Ouro Preto, 2010. 